\title{
TRATAMIENTO DE AGUAS RESIDUALES DE LA INDUSTRIA TEXTIL MEDIANTE COAGULACIÓN QUÍMICA ACOPLADA A PROCESOS FENTON INTENSIFICADOS CON ULTRASONIDO DE BAJA FRECUENCIA
}

\author{
Edison GILPAVAS*, Paula Eliana ARBELÁEZ-CASTAÑO, José David MEDINA-ARROYAVE y \\ Carlos Mario GÓMEZ-ATEHORTUA
}

Universidad Escuela de Administración, Finanzas y Tecnología (EAFIT), Carrera 49 núm. 7 Sur-50, Avenida Las Vegas, Medellín, Colombia

* Autor de correspondencia; egil@eafit.edu.co

(Recibido enero 2017; aceptado junio 2017)

Palabras clave: procesos avanzados de oxidación, sonoquímica, ultrasonido, Box-Behnken, optimización

\section{RESUMEN}

En la presente investigación se evaluó el tratamiento secuencial de coagulación química (CQ) seguida de los procesos avanzados de oxidación (PAO) Fenton $\left(\mathrm{H}_{2} \mathrm{O}_{2} / \mathrm{Fe}^{2+}\right.$ ) o foto-Fenton $\left(\mathrm{UV} / \mathrm{H}_{2} \mathrm{O}_{2} / \mathrm{Fe}^{2+}\right)$ intensificados con radiación de ondas de ultrasonido (US) de baja frecuencia. La optimización del proceso de pretratamiento con CQ, mediante la prueba de jarras, mostró que una dosis óptima de coagulante $\left(\mathrm{Al}_{2}\left[\mathrm{SO}_{4}\right]_{3}\right)$ de $800 \mathrm{mg} / \mathrm{L}$, remueve $99 \%$ de la turbidez y $53 \%$ de la demanda química de oxígeno (DQO). El clarificado resultante se empleó para la evaluación de los PAO. Mediante el análisis estadístico del diseño de experimentos de superficie de respuesta Box-Behnken se estableció que las condiciones óptimas de operación para el proceso $\mathrm{US} / \mathrm{H}_{2} \mathrm{O}_{2} / \mathrm{Fe}^{2+}$ fueron: $1 \mathrm{mM}$ de $\mathrm{Fe}^{2+}, 14 \mathrm{mM}$ de $\mathrm{H}_{2} \mathrm{O}_{2}$ y $\mathrm{pH}$ 3. En estas condiciones de operación el proceso de $\mathrm{CQ}$ acoplado con el proceso $\mathrm{US} / \mathrm{H}_{2} \mathrm{O}_{2} / \mathrm{Fe}^{2+}$ removió alrededor de $82 \%$ de la DQO en tanto que al acoplarlo con el proceso US/UV/ $\mathrm{H}_{2} \mathrm{O}_{2} / \mathrm{Fe}^{2+}$ alcanzó $95 \%$ de eliminación en 90 min de reacción. El uso de ondas de ultrasonido incrementa la eficiencia del proceso alrededor de $10 \%$. Durante el proceso también se monitorearon la mineralización de los contaminantes y el consumo de $\mathrm{H}_{2} \mathrm{O}_{2}$.

Key words: avanced oxidation process, sonochemistry, ultrasound, Box-Behnken, optimization

\begin{abstract}
The present study evaluates the sequential treatment of chemical coagulation (CC) followed by the advanced oxidation process (AOP) of Fenton $\left(\mathrm{H}_{2} \mathrm{O}_{2} / \mathrm{Fe}^{2+}\right)$ or photoFenton $\left(\mathrm{UV} / \mathrm{H}_{2} \mathrm{O}_{2} / \mathrm{Fe}^{2+}\right)$ intensified by low frequency ultrasound. Optimization of the pretreatment step through $\mathrm{CC}$ by jar test showed that an optimal coagulant $\left(\mathrm{Al}_{2}\left[\mathrm{SO}_{4}\right]_{3}\right)$ dose of $800 \mathrm{mg} / \mathrm{L}$ removed $99 \%$ of turbidity and $53 \%$ of the chemical oxygen demand (COD). The resulting supernatant is used for the AOP evaluation. The statistical analysis of a Box-Behnken response surface design showed that optimal conditions to carry out the $\mathrm{US} / \mathrm{H}_{2} \mathrm{O}_{2} / \mathrm{Fe}^{2+}$ process are: $1 \mathrm{mM}$ de $\mathrm{Fe}^{2+}, 14 \mathrm{mM}$ de $\mathrm{H}_{2} \mathrm{O}_{2}$ and $\mathrm{pH}$ 3. Under these conditions the $\mathrm{CC}$ coupled to the $\mathrm{US} / \mathrm{H}_{2} \mathrm{O}_{2} / \mathrm{Fe}^{2+}$ process removes $82 \%$ of the COD of the supernatant while the US/UV/ $\mathrm{H}_{2} \mathrm{O}_{2} / \mathrm{Fe}^{2+}$ process eliminates $95 \%$
\end{abstract}


after 90 min of reaction. The use of ultrasound waves inducing an increase of $10 \%$ in the process efficiency. The mineralization of pollutants during treatment as well as the $\mathrm{H}_{2} \mathrm{O}_{2}$ consumption were monitored.

\section{INTRODUCCIÓN}

La industria textil requiere gran cantidad de agua para sus procesos productivos. De 100 a $200 \mathrm{~L}$ de agua son necesarios para producir un kilogramo de productos textiles (Carvalho et al. 2016). El agua residual resultante está altamente contaminada debido a la presencia de colorantes, surfactantes, sales inorgánicas y distintos compuestos químicos empleados en el proceso productivo (Buscio et al. 2015). Los valores reportados para la demanda química de oxígeno (DQO) son de alrededor de 900 a $5000 \mathrm{mg} / \mathrm{L}$ y la demanda bioquímica de oxígeno $\left(\mathrm{DBO}_{5}\right)$ - con relaciones de biodegradabilidad $\left(\mathrm{DBO}_{5} / \mathrm{DQO}\right)$ - son inferiores a 0.15 (Cheshmeh y Safari 2016, Naje et al. 2016, Yurtsever et al. 2016). Por lo tanto, su descarga a cuerpos de agua, sin el tratamiento adecuado, es un riesgo para la calidad de los recursos hídricos, que son cada vez más escasos debido a la creciente población y demanda de agua (Doumic et al. 2015). Los tratamientos de aguas residuales textiles convencionales involucran la coagulación química con sales de hierro o aluminio combinada con procesos biológicos o adsorción (Verma et al. 2012, Vedrenne et al. 2012). El proceso de coagulación es eficiente para la remoción de coloides y sólidos suspendidos; diferentes estudios reportan que este tipo de contaminantes representan alrededor de $50 \%$ de la DQO total de un efluente textil (Jorfi et al. 2016, GilPavas et al. 2017). Por consiguiente, se emplea generalmente como etapa de pretratamiento, de ahí que sea necesario efectuar un tratamiento posterior con el objeto de eliminar los contaminantes remanentes y satisfacer la legislación ambiental. Después del proceso de coagulación, los compuestos orgánicos remanentes en este tipo de efluentes son compuestos con estructuras aromáticas complejas y detergentes con alta solubilidad en agua, resistentes a la degradación mediante procesos biológicos (Naje et al. 2016). Esto implica que los métodos convencionales son insuficientes y se requiere buscar alternativas eficientes para el tratamiento de este tipo de aguas residuales.

Los tratamientos alternativos que han sido objeto de estudio en las últimas décadas son los procesos avanzados de oxidación (PAO), como los procesos Fenton (F) y foto-Fenton (FF). Se ha encontrado que estos procesos son altamente eficientes para la degradación de colorantes presentes en aguas residuales de la industria textil (ARIT) (San Pedro-Cedillo et al. 2015, Doumic et al. 2015, Dias et al. 2016, GilPavas et al. 2017). Los procesos Fenton se basan en la generación de especies químicas altamente oxidantes, principalmente el radical hidroxilo $\left({ }^{\bullet} \mathrm{OH}\right)$ producido mediante la descomposición de $\mathrm{H}_{2} \mathrm{O}_{2}$ en presencia de $\mathrm{Fe}^{2+}$ como catalizador. Los mecanismos de reacción se encuentran ampliamente explicados en otros artículos publicados (Malato et al. 2009).

El tratamiento mediante ultrasonido (US) de baja frecuencia $(<50 \mathrm{kHz})$ se ha empleado recientemente para intensificar los procesos $\mathrm{F}$ (US/F) y FF (US/FF) (Wang y Shih 2015, Ammar 2016, Cai et al. 2016). El US se basa en el fenómeno de cavitación acústico, que ocurre dentro de un líquido por la aplicación de frecuencias de ultrasonido. La cavitación forma burbujas que son sometidas a una serie de ciclos de expansión y compresión, antes de colapsar violentamente (Papoutsakis et al. 2015). La cavitación acústica mejora los procesos de transferencia de masa y dispersión de las partículas de catalizador, a la vez que incrementa la disponibilidad de sitios activos para la reacción (Banerjee et al. 2012). Además, en los procesos US/F y US/FF, la irradiación US promueve la rápida regeneración de iones de $\mathrm{Fe}^{2+}$ y aumenta la producción de radicales ${ }^{\bullet} \mathrm{OH}$, sin consumo de $\mathrm{H}_{2} \mathrm{O}_{2}$. Los mecanismos de reacción se describen en las Ecs. (1) y (2) (Bagal y Gogate 2014).

$$
\begin{aligned}
& \mathrm{Fe}^{2+}+\mathrm{H}_{2} \mathrm{O}_{2} \stackrel{\text { Ondas }}{\longrightarrow} \mathrm{Fe}^{3+}+\mathrm{HO}^{\circ}+\mathrm{HO}^{-} \\
& \mathrm{Fe}^{3+}+\mathrm{H}_{2} \mathrm{O} \stackrel{\text { Ondas }}{\longrightarrow} \mathrm{Fe}^{2+}+\mathrm{HO}^{\cdot}+\mathrm{H}^{+}
\end{aligned}
$$

Esta investigación tiene como objetivo evaluar una metodología sistemática para el tratamiento de aguas residuales de una industria textil mediante los procesos secuenciales de coagulación-floculación, seguido de un PAO (US/F, US/FF). Según el conocimiento de los autores, ningún estudio ha evaluado el proceso US/F precedido de coagulación química como pretratamiento para aguas residuales industriales "reales" de una empresa textil. Tampoco se ha establecido el efecto de las diferentes variables de 
operación de los procesos Fenton intensificados con radiación US de baja frecuencia ni se ha determinado el efecto sinérgico de las ondas de US al combinarse con este tipo de procesos para el tratamiento de aguas residuales.

\section{MATERIALES Y MÉTODOS}

\section{Materiales}

Las muestras de agua residual textil se tomaron, al final del proceso, en el tanque de homogenización de efluentes residuales - el cual almacena los efluentes resultantes de los procesos de lavado, desengomado y tinturado - de una empresa del sector textil dedicada a la fabricación de pantalones localizada en Medellín, Colombia. Las muestras de agua residual fueron refrigeradas $\left(<4{ }^{\circ} \mathrm{C}\right)$ durante su almacenamiento y transporte, para evitar la degradación de compuestos, de acuerdo con los procedimientos estándar (APHA 2012). La caracterización inicial del agua se realizó por triplicado, obteniéndose una variación en los resultados inferior a $5 \%$. Los valores promedio obtenidos se presentan en el cuadro I, junto con los límites permisibles establecidos en la legislación colombiana, de acuerdo con la Resolución 0631 de 2015. $\mathrm{El} \mathrm{FeSO} \cdot 7 \mathrm{H}_{2} \mathrm{O}(99 \% \mathrm{w} / \mathrm{w})$ y el $\mathrm{H}_{2} \mathrm{O}_{2}(30 \%$ $\mathrm{w} / \mathrm{w})$ (Panreac), se usaron sin modificación en los procesos F y FF. Para la coagulación química se empleó $\mathrm{Al}_{2}\left(\mathrm{SO}_{4}\right)_{3} \cdot 18 \mathrm{H}_{2} \mathrm{O}$ al $99 \%$ de pureza (Panreac). El pH se ajustó con $\mathrm{H}_{2} \mathrm{SO}_{4}$ al $99.1 \%$ (Merk). Las diluciones requeridas se prepararon con agua ultra pura del sistema Milli-Q (resistividad: $18 \mathrm{~m} \Omega \cdot \mathrm{cm}$ ).

\section{Métodos analíticos}

Para el seguimiento de la degradación del índigo y materia orgánica, se utilizó la técnica de espectrofotometría (espectrofotómetro, Nanocolor de Macherey-Nagel). Las concentraciones se determinaron a partir de sus características de absorbancia en el UV-Vis (200-700 $\mathrm{nm}$ ) con una celda de $1 \mathrm{~cm}$ de paso óptico y se midieron con base en la variación de la longitud de onda de máxima absorbancia $(660 \mathrm{~nm})$. El procedimiento empleado para medir la DQO fue el método estándar de reflujo cerrado (método 5220D, Kit test 0-26, DQO 160, Macherey-Nagel). La medición de la turbidez se realizó de acuerdo con el método 2130-B. La medición del carbono orgánico total (COT) se llevó a cabo usando el método estándar de oxidación húmeda con medición colorimétrica (método 5310D, Kit test 0-94, COT 60, Macherey-Nagel). Para la medición de la $\mathrm{DBO}_{5}$ se empleó el método estándar respirométrico 5210D (APHA 2012). En estos procedimientos se utilizó un termorreactor Spectrocuant TR 420 Merck y el equipo Nanocolor 500D Macherey-Nagel. El pH y la conductividad de las muestras se determinaron con el equipo multiparamétrico (pHmetro y conductímetro) Thermo Scientific Orion 5 Stars. La concentración de $\mathrm{H}_{2} \mathrm{O}_{2}$ se cuantificó mediante titulación yodométrica con $\mathrm{Na}_{2} \mathrm{~S}_{2} \mathrm{O}_{3}$ al $99 \%$ de pureza (Merk). Para evitar la interferencia de $\mathrm{H}_{2} \mathrm{O}_{2}$ durante las mediciones de la DQO, el $\mathrm{H}_{2} \mathrm{O}_{2}$ residual se inhibió con $\mathrm{MnO}_{2}$, grado reactivo $90 \% \mathrm{w} / \mathrm{w}$ (Sigma-Aldrich).

\section{Pretratamiento de coagulación-floculación}

El proceso de CQ se realizó en un equipo para prueba de jarras (Centricol) equipado con seis compartimentos iguales de $2 \mathrm{~L}$ de capacidad. La prueba se efectuó realizó de acuerdo con el procedimiento estándar ASTM D2035:2008, que consiste en tres etapas: 1) mezcla rápida simultánea de dosis de 400 , $500,600,700,800$ y $900 \mathrm{mg} / \mathrm{L}$ de $\mathrm{Al}_{2}\left(\mathrm{SO}_{4}\right)_{3} \cdot 18 \mathrm{H}_{2} \mathrm{O}$, con agitación de $500 \mathrm{rpm}$ durante $1 \mathrm{~min}$ para la disolución uniforme del coagulante; 2) agitación lenta a $20 \mathrm{rpm}$ durante 20 min para promover la formación de flóculos, y 3) 15 min de sedimentación sin agitación. El proceso de coagulación se efectuó al $\mathrm{pH}$ natural del agua de acuerdo con ensayos preliminares. Al final del proceso se midió la DQO, el color y la turbidez residual del sobrenadante para cada una de las dosis evaluadas. El ensayo que reportó la menor concentración de colorante, DQO y turbidez residual se seleccionó para efectuar el pretratamiento.

CUADRO I. CARACTERÍSTICAS FISICAS Y QUÍMICAS DEL AGUA RESIDUAL TEXTIL

\begin{tabular}{|c|c|c|c|c|c|c|c|c|}
\hline Parámetro & $\mathrm{pH}$ & $\begin{array}{l}\text { Conductividad } \\
(\mu \mathrm{S} / \mathrm{cm})\end{array}$ & $\begin{array}{l}\text { Turbidez } \\
\text { (UNT) }\end{array}$ & $\begin{array}{l}\text { DQO } \\
(\mathrm{mg} / \mathrm{L})\end{array}$ & $\begin{array}{c}\mathrm{COT} \\
(\mathrm{mg} / \mathrm{L})\end{array}$ & $\begin{array}{l}\mathrm{DBO}_{5} \\
(\mathrm{mg} / \mathrm{L})\end{array}$ & $\underset{(\mathrm{mg} / \mathrm{L})}{\mathrm{ST}}$ & $\mathrm{DBO}_{5} / \mathrm{DQO}$ \\
\hline Agua residual & 7.06 & 4520 & 308 & 420 & 124 & 36.5 & 1341 & 0.087 \\
\hline Valores permisibles (Res. 0631) & $6-9$ & - & - & 360 & - & 200 & - & - \\
\hline
\end{tabular}

pH: potencial de hidrógeno; conductividad: capacidad de conducción eléctrica; UNT: unidades nefelométricas de turbidez; DQO: demanda química de oxígeno; COT: carbono orgánico total; $\mathrm{DBO}_{5}$ : demanda bioquímica de oxígeno; ST: sólidos totales; DBO 5 /DQO: relación de biodegrabilidad; Res.: resolución del Ministerio de Medio Ambiente, Colombia, marzo de 2015 


\section{Sistema de reacción para US/F y US/FF}

Una vez efectuado el pretratamiento de CQ, el PAO comienza con la adición de las dosis correspondientes de $\mathrm{FeSO}_{4} \cdot 7 \mathrm{H}_{2} \mathrm{O}$ en estado sólido y $\mathrm{H}_{2} \mathrm{O}_{2}$ en estado líquido al agua residual textil. La mezcla se agita mecánicamente a $500 \mathrm{rpm}$ con el fin de homogenizar la solución y mejorar fenómenos de transferencia de masa. Para los procesos F y FF, el reactor se ubica en un baño de ultrasonido BRANSON 1800 con frecuencia de operación de $40 \mathrm{kHz}$. El proceso $\mathrm{F}$ se realizó por lotes usando un reactor de borosilicato de $150 \mathrm{ml}$ de capacidad. Para los experimentos de FF se emplea una lámpara tubular Black Light con longitud de onda característica $\lambda=365 \mathrm{~nm}, 15 \mathrm{~mm}$ de diámetro y $225 \mathrm{~mm}$ de longitud (modelo F6T5/ BL Philips, $6 \mathrm{~W}$, flujo fotónico de $1.47 \times 10^{19} 1 / \mathrm{m}^{2} / \mathrm{s}$, flujo radiante $0.5 \mathrm{~mW} / \mathrm{cm}^{2}$ ).

\section{Modelo estadístico y diseño de experimentos}

Las variables de operación para la optimización del proceso US/F se seleccionaron de acuerdo con la revisión bibliográfica y experiencia del grupo de investigación. El pH es uno de los factores significativos para los procesos Fenton; el hierro tiende a formar complejos insolubles con el agua a $\mathrm{pH}$ por encima de 4 (Malato et al. 2009), lo que reduce la cantidad de hierro disponible como catalizador, disminuyendo la eficiencia del proceso. Sin embargo, se ha reportado que el US permite realizar los procesos Fenton satisfactoriamente a $\mathrm{pH}$ superiores. Papoutsakis et al. (2015) lograron la remoción completa del fenol con el proceso US/F a pH de 5.5. En esta investigación el intervalo de operación para el $\mathrm{pH}$ fue de 3 a 5 . Otro factor significativo es la $\left[\mathrm{Fe}^{2+}\right]$, que cataliza la descomposición del $\mathrm{H}_{2} \mathrm{O}_{2}$, generando las especies reactivas oxidantes que promueven la reacción Fenton (Blanco et al. 2014). Adicionalmente, las vibraciones US pueden suscitar la regeneración de $\mathrm{Fe}^{2+}$ (Sheydaei y Khataee 2015), lo cual determina que el proceso US/F requiera menor $\left[\mathrm{Fe}^{2+}\right]$ o menos tiempo de operación que en el proceso $\mathrm{F}$ tradicional. El intervalo de operación para la $\left[\mathrm{Fe}^{2+}\right]$ fue de 0 a 1 $\mathrm{mM}$, debido a que diferentes estudios demuestran que a $\left[\mathrm{Fe}^{2+}\right]>1 \mathrm{mM}$ se generan incrementos muy leves en la eficiencia del proceso (Malato et al. 2009). El último factor operacional considerado fue $\mathrm{la}\left[\mathrm{H}_{2} \mathrm{O}_{2}\right]$. En los procesos US/F, las ondas de US promueven la generación adicional de radicales ${ }^{\bullet} \mathrm{OH}$ a partir de la hidrolisis del agua, sin consumo de $\mathrm{H}_{2} \mathrm{O}_{2}$ (Ammar 2016). Con base en trabajos previos (GilPavas et al. 2015), se estableció la $\left[\mathrm{H}_{2} \mathrm{O}_{2}\right]$ entre 0 y $19.4 \mathrm{mM}$. Se consideró el nivel inferior 0 para la $\left[\mathrm{Fe}^{2+}\right]$ y $\left[\mathrm{H}_{2} \mathrm{O}_{2}\right]$, con el fin de evaluar en el DDB los procesos $\mathrm{US} / \mathrm{H}_{2} \mathrm{O}_{2}$ y US/ $/ e^{2+}$. El tiempo de reacción para el proceso fue de $30 \mathrm{~min}$.

Se seleccionó el diseño experimental de metodología de superficie de respuesta (MSR) Box-Behnken (DBB) con tres puntos centrales, a fin de establecer el efecto de los factores operacionales seleccionados y sus interacciones sobre el porcentaje de degradación de materia orgánica. El modelo DBB es útil para determinar las condiciones óptimas de operación, debido a que permite: 1) la estimación de los parámetros de un polinomio de segundo orden, el cual describe la variable respuesta en función de las condiciones de operación; 2) la detección del ajuste del modelo generado mediante los coeficientes de determinación $\mathrm{R}^{2}$ y $\mathrm{R}^{2}$ adj, y 3 ) la construcción de diseños secuenciales y en bloques. La MSR no se usa para establecer los mecanismos del proceso; su objetivo es la determinación de las condiciones de operación que maximicen o minimicen la variable respuesta de interés. Para el análisis estadístico se realizó, en primer lugar, un análisis de varianza, que junto con el diagrama de Pareto proporciona información acerca de las condiciones de operación más significativas en el proceso. Posteriormente se efectuaron diagramas de interacciones y superficies de respuestas, con el objeto de evaluar los efectos individuales e interacciones de cada uno de los factores. Los detalles de esta metodología se encuentran descritos en otros trabajos (GilPavas et al. 2014). El cuadro II presenta los factores de operación y niveles evaluados en el DBB.

CUADRO II. VARIABLES (FACTORES) CON SUS NIVELES PARA EL DISEÑO BOX-BEHNKEN

\begin{tabular}{lccc}
\hline Variables & \multicolumn{3}{c}{ Factores codificados, $\mathrm{X}$} \\
\cline { 2 - 4 } & Nivel 1 & Nivel 2 & Nivel 3 \\
\hline $\mathrm{H}_{2} \mathrm{O}_{2}(\mathrm{mM})$ & 0 & 9.7 & 19.4 \\
$\mathrm{Fe}^{2+}(\mathrm{mM})$ & 0 & 0.5 & 1 \\
$\mathrm{pH}$ & 3 & 4 & 5 \\
\hline
\end{tabular}

\section{RESULTADOS Y DISCUSIÓN}

\section{Pretratamiento de coagulación-floculación}

El principal propósito de aplicar el proceso CQ como pretratamiento es alcanzar una alta remoción de los sólidos suspendidos totales (SST) (Silva et al. 2016). La turbidez residual es una medida indirecta de la cantidad de SST, cuya medición es fácil y rápida (APHA 2012) y proporciona una idea precisa de la eficiencia del proceso de coagulación. En este proceso, 


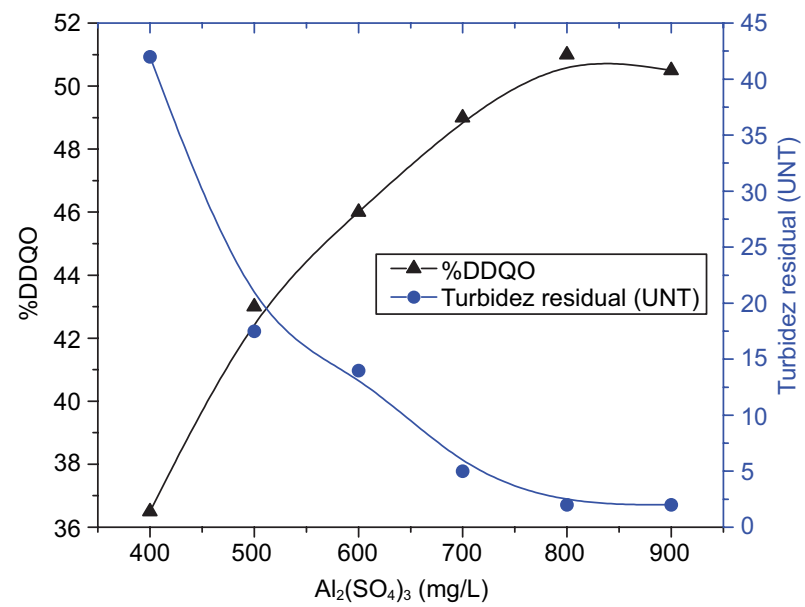

Fig. 1. Prueba de jarras: dosis de coagulante vs. turbidez* y $\%$ de degradación de la demanda química de oxígeno (\% DDQO). UNT: unidades nefelométricas de turbidez

las sales de aluminio inducen iones positivamente cargados a la mezcla, los cuales reducen el grado de repulsión electrostática entre partículas coloidales; esto permite que las fuerzas de atracción superen las de repulsión, lo cual rompe la dispersión y hace que la mezcla se flocule (Amor et al. 2015). El objetivo de la prueba de jarras fue determinar la dosis óptima de coagulante para efectuar el proceso. La figura 1 muestra la evolución de la turbidez residual y el porcentaje de disminución de la demanda química de oxígeno (\% DDQO), empleando dosis de 400 a $900 \mathrm{mg} / \mathrm{L}$ de $\mathrm{Al}_{2}\left(\mathrm{SO}_{4}\right)_{3}$ a $\mathrm{pH}$ natural (7). Se observa que los incrementos en las dosis de coagulante generan una reducción en la turbidez residual y un aumento en el \% de DDQO hasta los $800 \mathrm{mg} / \mathrm{L}$. A partir de este punto, mayores dosis de coagulante no promueven algún efecto sobre la eficiencia del proceso, por lo tanto la dosis óptima para efectuar el pretratamiento se fijó en $800 \mathrm{mg} / \mathrm{L}$.

Mediante el proceso de coagulación, la DQO se reduce de 420 a $195 \mathrm{mg} / \mathrm{L}$ y la $\mathrm{DBO}_{5}$ pasa de 36.5 a $25.5 \mathrm{mg} / \mathrm{L}$, lo que indica que los sólidos suspendidos representan alrededor de la mitad de la materia orgánica presente en la muestra. La otra mitad está conformada por compuestos solubles resistentes a la coagulación que requieren procesos adicionales para su eliminación. La relación $\mathrm{DBO}_{5} / \mathrm{DQO}$ al final de la etapa de coagulación es igual a 0.2 , lo cual significa que los tratamientos biológicos serán ineficientes en la remoción de la carga orgánica remanente y que procesos como los PAO son idóneos en estos escenarios (Rodríguez et al. 2002, GilPavas et al. 2017). La etapa de pretratamiento fue $100 \%$ eficiente para la remoción del colorante (principalmente índigo y trazas de otros colorantes) y no reportó absorbancia en el espectro visible (350 a $700 \mathrm{~nm}$ ). Por lo tanto, el porcentaje de degradación de color no se consideró como variable de respuesta para los procesos posteriores. Al usar 800 $\mathrm{mg} / \mathrm{L}$ de coagulante, el $\mathrm{pH}$ final fue 5 y se consideró como uno de los factores a evaluar en el diseño de optimización Box-Behnken para el proceso US/F.

\section{Optimización del proceso sono-Fenton (US/F)}

Las condiciones de operación de los 15 ensayos del DBB se presentan en el cuadro III junto con los resultados obtenidos. En la primera columna se presentan las mediciones del \% de DDQO (Y $\left.\mathrm{Y}_{\text {exp }}\right)$ para los ensayos experimentales y en la segunda columna los resultados predichos ( $\left.\mathrm{Y}_{\text {pred }}\right)$ por el modelo polinómico de segundo orden (Ec. 3), cuyos coeficientes de regresión se obtuvieron a partir del análisis estadístico y se emplean para realizar la optimización del proceso. Se observa que el mayor \% de DDQO fue de $46.15 \%$, y corresponde a $\mathrm{pH} 3,\left[\mathrm{Fe}^{2+}\right]: 1 \mathrm{mM}$ y $\left[\mathrm{H}_{2} \mathrm{O}_{2}\right]: 9.7 \mathrm{mM}$.

$$
\begin{aligned}
\% D D Q O & =-19.8283+(3.32167 * p H)+ \\
& \left(21.7033 * \mathrm{Fe}^{2+}\right)+\left(7.24863 * \mathrm{H}_{2} \mathrm{O}_{2}\right) \\
& +\left(0.566667 * p \mathrm{H}^{2}\right)-\left(7.05 * p H * \mathrm{Fe}^{2+}\right) \\
& -\left(1.12371 * p H * \mathrm{H}_{2} \mathrm{O}_{2}\right)+(11.8267 \\
& \left.*\left(\mathrm{Fe}^{2+}\right)^{2}\right)+\left(1.15361 * \mathrm{Fe}^{2+} * \mathrm{H}_{2} \mathrm{O}_{2}\right) \\
& -\left(0.121834 *\left(\mathrm{H}_{2} \mathrm{O}_{2}\right)^{2}\right)
\end{aligned}
$$

\begin{tabular}{|c|c|c|c|c|}
\hline \multirow{2}{*}{$\begin{array}{l}\mathrm{H}_{2} \mathrm{O}_{2} \\
{[\mathrm{mM}]}\end{array}$} & \multicolumn{2}{|c|}{ Variables } & \multicolumn{2}{|c|}{$\begin{array}{l}\text { Resultados } \\
\text { (\% de DDQO) }\end{array}$} \\
\hline & {$[\mathrm{mM}]$} & & $Y_{\exp }$ & $Y_{\text {pred }}$ \\
\hline 19.4 & 1 & 4 & 33.33 & 37.97 \\
\hline 9.7 & 0.5 & 4 & 21.79 & 23.07 \\
\hline 0 & 0.5 & 3 & 0.00 & 1.44 \\
\hline 0 & 0 & 4 & 7.69 & 3.04 \\
\hline 0 & 1 & 4 & 5.13 & 7.85 \\
\hline 9.7 & 0.5 & 4 & 24.36 & 23.07 \\
\hline 19.4 & 0 & 4 & 12.82 & 10.09 \\
\hline 9.7 & 1 & 3 & 46.15 & 44.86 \\
\hline 0 & 0.5 & 5 & 3.85 & 7.21 \\
\hline 9.7 & 0.5 & 4 & 23.08 & 23.07 \\
\hline 9.7 & 0 & 3 & 15.38 & 21.47 \\
\hline 19.4 & 0.5 & 3 & 42.31 & 38.94 \\
\hline 9.7 & 0 & 5 & 14.10 & 15.38 \\
\hline 9.7 & 1 & 5 & 30.77 & 24.67 \\
\hline 19.4 & 0.5 & 5 & 2.56 & 4.00 \\
\hline
\end{tabular}

CUADRO III. RESULTADOS EXPERIMENTALES (YEXP) Y PREDICHOS (Y PRED) PARA LA DEGRADACIÓN DE LA DEMANDA QUÍMICA DE OXÍGENO (\% DE DDQO) MEDIANTE EL TRATAMIENTO US/F 
CUADRO IV. ANÁLISIS DE LA VARIANZA PARA LA DEGRADACIÓN DE DEMANDA QUÍMICA DE OXÍGENO (\% DDQO) EN FUNCIÓN DE $\left[\mathrm{Fe}^{2+}\right],\left[\mathrm{H}_{2} \mathrm{O}_{2}\right] \mathrm{Y} \mathrm{pH}$

\begin{tabular}{lccccc}
\hline \multicolumn{1}{c}{ Fuente } & $\begin{array}{c}\text { Suma de } \\
\text { cuadrados }\end{array}$ & $\begin{array}{c}\text { Grados de } \\
\text { libertad }\end{array}$ & $\begin{array}{c}\text { Cuadrado } \\
\text { medio }\end{array}$ & Razón-F & Valor $\mathrm{p}^{*}$ \\
\hline $\mathrm{A}: \mathrm{Fe}^{2+}$ & 534.482 & 1 & 534.482 & 16.13 & $\mathbf{0 . 0 1 0 2}$ \\
$\mathrm{B}: \mathrm{H}_{2} \mathrm{O}_{2}$ & 690.99 & 1 & 690.99 & 20.85 & $\mathbf{0 . 0 0 6}$ \\
$\mathrm{C}: \mathrm{pH}$ & 345.319 & 1 & 345.319 & 10.42 & $\mathbf{0 . 0 2 3 3}$ \\
$\mathrm{AA}$ & 34.1883 & 1 & 34.1883 & 1.03 & 0.3563 \\
$\mathrm{AB}$ & 133.056 & 1 & 133.056 & 4.02 & 0.1014 \\
$\mathrm{AC}$ & 49.7025 & 1 & 49.7025 & 1.5 & 0.2752 \\
$\mathrm{BB}$ & 477.925 & 1 & 477.925 & 14.42 & $\mathbf{0 . 0 1 2 7}$ \\
$\mathrm{BC}$ & 475.24 & 1 & 475.24 & 14.34 & $\mathbf{0 . 0 1 2 8}$ \\
$\mathrm{CC}$ & 0.8522 & 1 & 0.8522 & 0.03 & 0.8789 \\
Error total & 165.674 & 5 & 33.1348 & & \\
Total (corr.) & 2933.73 & 14 & & & \\
$\mathrm{R}^{2}=94.3528$ & & & & & \\
$\mathrm{R}^{2}$ adj $=84.19$ & & & & & \\
\hline
\end{tabular}

*Los valores en negritas indican variables estadísticamente significativas $\mathrm{R}^{2}$ adj: coeficiente de regresión que indica el ajuste del modelo

\section{Análisis estadísticos}

El análisis de varianza se utilizó para establecer si las contribuciones de los factores operacionales y sus interacciones inducen un efecto significativo sobre la variable respuesta. Las pruebas se realizaron con un intervalo de confianza de $95 \%$ y un valor de $\mathrm{p} \leq 0.05$, lo cual implica que el factor produce una variación estadísticamente significativa sobre la variable respuesta (Montgomery 2005). El cuadro IV presenta el análisis de varianza para el \% de DDQO en función de los factores $\left[\mathrm{Fe}^{2+}\right],\left[\mathrm{H}_{2} \mathrm{O}_{2}\right]$ y $\mathrm{pH}$. De acuerdo con este análisis, cada uno de los factores individuales evaluados presentan un valor de $\mathrm{p}$ $\leq 0.05$, por lo tanto, son estadísticamente significativos en el proceso US/F. Adicionalmente, la interacción $\mathrm{pH}-\mathrm{H}_{2} \mathrm{O}_{2}$ y el termino cuadrado de $\mathrm{H}_{2} \mathrm{O}_{2}$ generan variaciones significativas sobre el $\%$ de DDQO. El hecho que los tres factores sean significativos, implica que influyen en la eficiencia del proceso. A continuación se realiza un análisis estadístico complementario para conocer la forma y magnitud de cada uno de los factores.

El diagrama de Pareto (Fig. 2) proporciona en forma gráfica información similar al cuadro IV, donde se muestran los resultados del análisis de varianza. En dicha figura, los factores o interacciones que sobrepasan la línea vertical son los que tienen un efecto significativo sobre la eficiencia del proceso. El diagrama de Pareto es útil para establecer si los factores producen un efecto directo o inversamente proporcional sobre la variable respuesta. En este caso la $\left[\mathrm{H}_{2} \mathrm{O}_{2}\right]$ y $\left[\mathrm{Fe}^{2+}\right]$



Fig. 2. Diagrama de Pareto para A: $\left[\mathrm{Fe}^{2+}\right], \mathrm{B}:\left[\mathrm{H}_{2} \mathrm{O}_{2}\right], \mathrm{C}$ : $\mathrm{pH}$ y sus interacciones

tienen signo $(+)$, lo que significa que un aumento en su concentración produce un incremento sobre el $\%$ de DDQO, en tanto que el $\mathrm{pH}$ presenta signo (-), evidenciando que en el intervalo evaluado valores menores de $\mathrm{pH}$ producen eficiencias mayores en el tratamiento.

De acuerdo con el análisis estadístico, el resultado obtenido para el $\mathrm{pH}$ concuerda con lo reportado por diversos autores respecto de que las mayores eficiencias se obtienen con $\mathrm{pH}$ cercanos a 3. Esto se atribuye a que los complejos de hierro que se forman en el agua son más estables a $\mathrm{pH}$ bajos, mientras que a $\mathrm{pH}>4$ presentan muy baja solubilidad y se precipitan (Miralles-Cuevas et al. 2014. Por otro lado, el 
signo (+) encontrado para las $\left[\mathrm{H}_{2} \mathrm{O}_{2}\right]$ y $\left[\mathrm{Fe}^{2+}\right]$ también concuerda con lo esperado: a medida que se aumenta su concentración se incrementa la disponibilidad de reactivos para efectuar la reacción Fenton y por tanto se obtienen mayores eficiencias de degradación. Otras investigaciones (Gernjak et al. 2006, Malato et al. 2009) han reportado que aumentos en las dosis de los reacti-

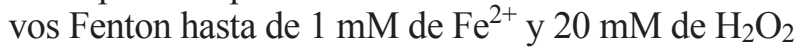
conducen a un incremento en la eficiencia del proceso.

\section{Selección de las condiciones óptimas mediante análisis de interacciones y superficie de respuesta}

Cuando dos o más factores se involucran en un diseño experimental, el análisis de interacciones permite encontrar si el efecto de cada uno de los factores sobre la variable respuesta es independiente o dependiente de la magnitud de los otros factores (Montgomery 2005). Además, proporciona información importante para la selección de las condiciones óptimas de operación. La figura 3a, $\mathbf{b}$ presentan los diagramas de interacciones entre los factores $\mathrm{pH}-\mathrm{H}_{2} \mathrm{O}_{2}$ y Fe${ }^{2+}-\mathrm{pH}$

La figura 3a muestra que en ausencia de $\mathrm{H}_{2} \mathrm{O}_{2}$ la degradación es mínima, mientras que con $19.4 \mathrm{mM}$ de $\mathrm{H}_{2} \mathrm{O}_{2}$ se obtienen los mayores \% de DDQO cuando el pH es cercano a 3. Esto se explica debido a que en las reacciones Fenton, el $\mathrm{H}_{2} \mathrm{O}_{2}$ se descompone catalizado por la presencia del $\mathrm{Fe}^{2+}$ para generar especies químicas oxidantes que degradan los contaminantes (Arslan-Alaton et al. 2009). En ausencia de $\mathrm{H}_{2} \mathrm{O}_{2}$ la producción de radicales ${ }^{\bullet} \mathrm{OH}$ no se inicia; por tanto, la materia orgánica presente no es degradada. Por el contrario, cuando se inyecta $\mathrm{H}_{2} \mathrm{O}_{2}$ se observa descomposición de materia orgánica, proceso que es más eficiente a pH 3. Al incrementar el $\mathrm{pH}$ el hierro tiende a precipitarse y la eficiencia del proceso disminuye (Malato et al. 2009).

En la figura 3b se observa que, en ausencia de $\mathrm{Fe}^{2+}$, la eficiencia del proceso disminuye significativamente; en estas condiciones, el proceso de degradación es producido únicamente por la descomposición del $\mathrm{H}_{2} \mathrm{O}_{2}$. Por otro lado, al emplear una dosis de 1 $\mathrm{mM}$ de $\mathrm{Fe}^{2+}$, en combinación con $\mathrm{H}_{2} \mathrm{O}_{2}$ y US, se efectúan las reacciones descritas en las Ecs. (1) y (2), donde el radical ${ }^{\bullet} \mathrm{OH}$ oxida la materia orgánica presente dando lugar al aumento en el \% de DDQO. De igual manera, en la figura $\mathbf{3 b}$ se observa que el pH 3 favorece la degradación de contaminantes, lo que se asocia a la mayor estabilidad de los iones $\mathrm{Fe}^{2+}$. Con base en los resultados obtenidos, se considera que el pH 3 es un parámetro óptimo para desarrollar el proceso. Valores inferiores de $\mathrm{pH}$ no se toman en cuenta debido a que requieren cantidades altas de ácido e implicarían altos costos de operación.
Los diagramas de superficie de respuesta son útiles para determinar el efecto simultáneo de dos factores sobre la variable respuesta. Se construyeron a partir del polinomio descrito en la Ec. (3), dejando constante el $\mathrm{pH}$ a un valor de 3 , ya que éste presenta las mayores eficiencias. En la figura 4 se observa que en ausencia de $\mathrm{Fe}^{2+}$ y $\mathrm{H}_{2} \mathrm{O}_{2}$, la degradación de materia orgánica es cercana a cero, lo cual permite concluir que las ondas de US de baja frecuencia por sí solas son insuficientes para producir especies oxidantes que descompongan los contaminantes presentes en el agua. A medida que aumentan las $\left[\mathrm{Fe}^{2+}\right]$ y $\left[\mathrm{H}_{2} \mathrm{O}_{2}\right]$, se incrementa la eficiencia del proceso. En el caso del $\mathrm{H}_{2} \mathrm{O}_{2}$ se observa que el incremento que genera sobre el \% de DDQO no es lineal: una dosis de $14 \mathrm{mM}$ es suficiente para alcanzar eliminaciones de la DQO

(a)

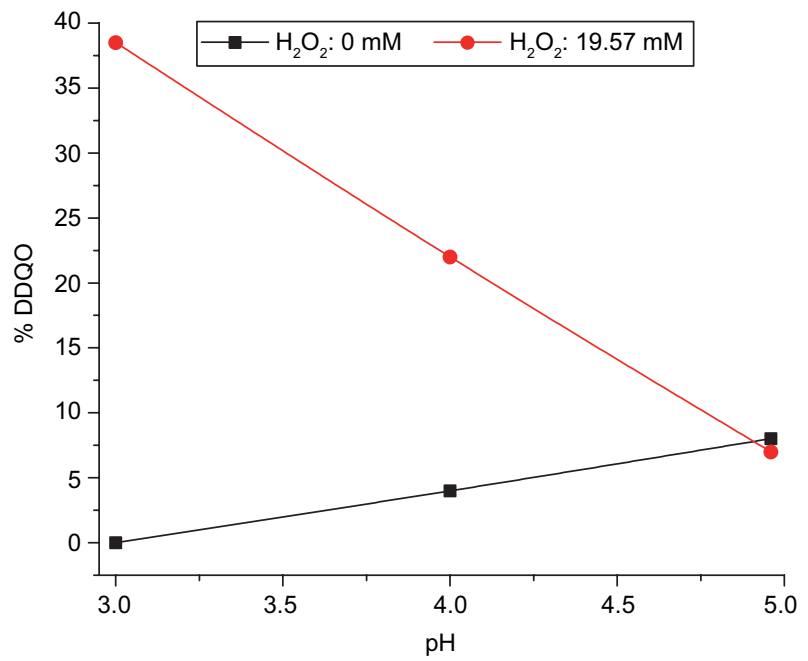

(b)

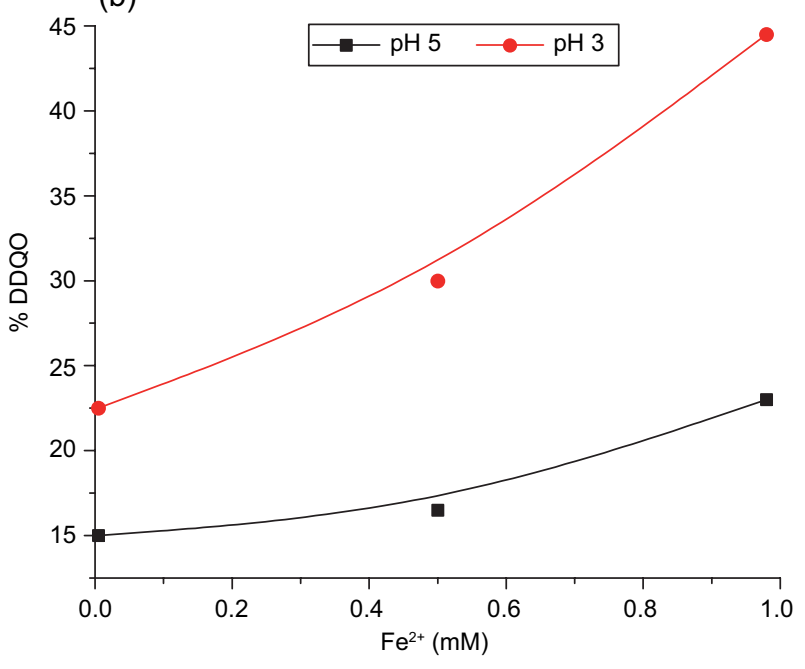

Fig. 3. Diagramas de interacciones para la degradación de demanda química de oxígeno (\% DDQO) 




Fig. 4. Diagrama de superficie de respuesta para la degradación de demanda química de oxígeno (\% DDQO)

mayores que $45 \%$ en 30 min de reacción; por encima de esta concentración, el incremento en el \% de DDQO es más lento. Esto se explica porque en presencia de una $\left[\mathrm{H}_{2} \mathrm{O}_{2}\right]$ elevada, los radicales ${ }^{\bullet} \mathrm{OH}$ tienden a recombinarse para formar $\mathrm{H}_{2} \mathrm{O}_{2} \mathrm{y}$ otras especies químicas con menor poder oxidante (Doumic et al. 2015). En el caso del $\mathrm{Fe}^{2+}$ se observa una relación lineal con el \% de DDQO para el intervalo evaluado, lo cual implica que sería posible encontrar mayores eficiencias con $\left[\mathrm{Fe}^{2+}\right]>1 \mathrm{mM}$. Sin embargo, involucraría altos costos de reactivos y estaría por encima del límite permisible por la legislación ambiental. El hierro se regenera durante la reacción (Malato et al. 2009), por lo que sería posible alcanzar mayores eficiencias con tiempos de reacción superiores a $30 \mathrm{~min}$.

Con base en el análisis estadístico se define que las condiciones óptimas para efectuar el proceso US/F y evaluar su comportamiento en el tiempo son: $\mathrm{pH}$ $3,1 \mathrm{mM}$ de $\left[\mathrm{Fe}^{2+}\right]$ y $14 \mathrm{mM}$ de $\left[\mathrm{H}_{2} \mathrm{O}_{2}\right]$.

En la figura 5a se presenta la evolución del \% de DDQO y agotamiento de $\mathrm{H}_{2} \mathrm{O}_{2}$, tanto para el proceso US/F como para los procesos US/FF, F y $\mathrm{FF}$, con el objetivo de analizar las contribuciones de los tratamientos individuales y el efecto sinérgico al emplear los procesos acoplados, en las condiciones experimentales seleccionadas con base en análisis estadístico. Es importante resaltar que en este caso se monitoreó la evolución de la DQO, COT y el barrido espectral durante 90 min de reacción; además, se incluyó la eficiencia de remoción que aportó el tratamiento preliminar CQ; por lo tanto, se registran mayores eficiencias que las reportadas en el diseño experimental. Se observa que el proceso US/F, analizado en el diseño experimental, logra $84 \%$ de eliminación de DQO en los 90 min de reacción, mientras que el proceso US/FF alcanza $95 \%$ de DDQO. En ambos procesos la radiación de ondas US incrementa alrededor de $10 \%$ la eficiencia del proceso, con relación a los procesos individuales; esto se debe al aumento en la producción de radicales ${ }^{\bullet} \mathrm{OH}$, de acuerdo con las Ecs. 1 y 2, así como al fenómeno de cavitación acústica que mejora los procesos de transferencia de masa (Banerjee et al. 2012). Los procesos en los que se incluye radiación UV son más eficientes debido a la regeneración de $\mathrm{Fe}^{3+}$ a Fe${ }^{2+}$, al tiempo que se producen más especies oxidantes (Gernjak et al. 2006). $\mathrm{El}$ agotamiento del $\mathrm{H}_{2} \mathrm{O}_{2}$ qeu se muestra en la figura 5a también proporciona una medida de la evolución de la reacción, debido a que se consume para oxidar los contaminantes presentes en la muestra.

La figura $5 \mathbf{b}$ presenta la evolución del porcentaje de degradación del carbono orgánico total (\% DCOT) a medida que avanza la reacción. EL COT proporciona una medida de la cantidad de carbono asociado a compuestos orgánicos en la muestra; por lo tanto, su reducción implica que éstos son mineralizados, es decir, la conversión a $\mathrm{CO}_{2}, \mathrm{H}_{2} \mathrm{O}$ y ácidos inorgánicos (GilPavas et al. 2017). Se observa que el proceso de CQ es eficiente para remover $40 \%$ del COT. El remanente corresponde a compuestos orgánicos solubles resistentes a la coagulación, como compuestos 


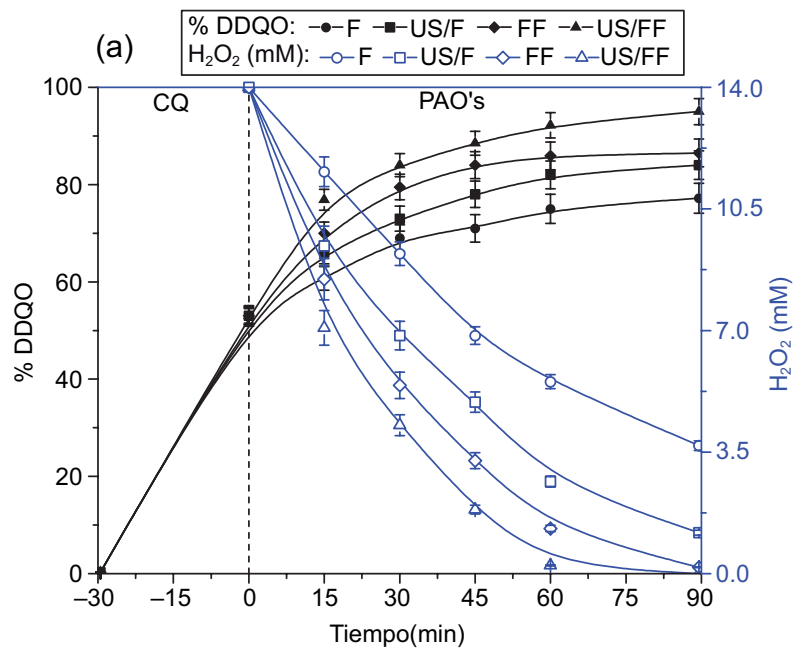

(b)

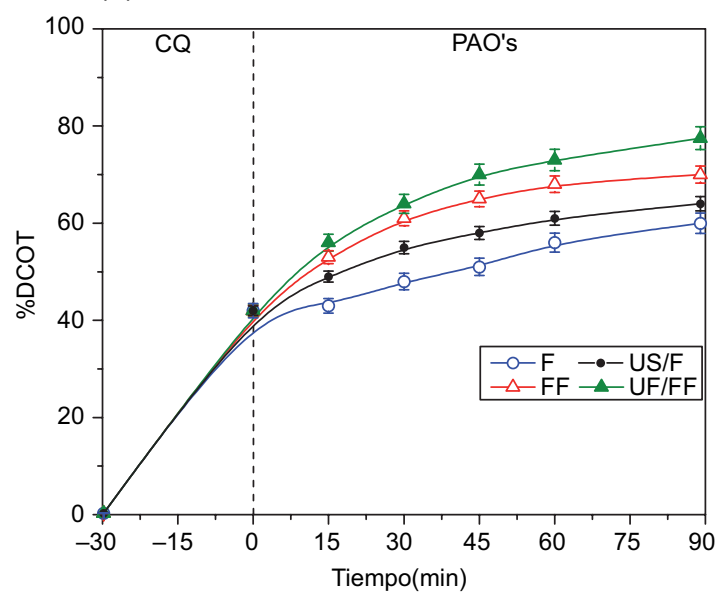

(c)

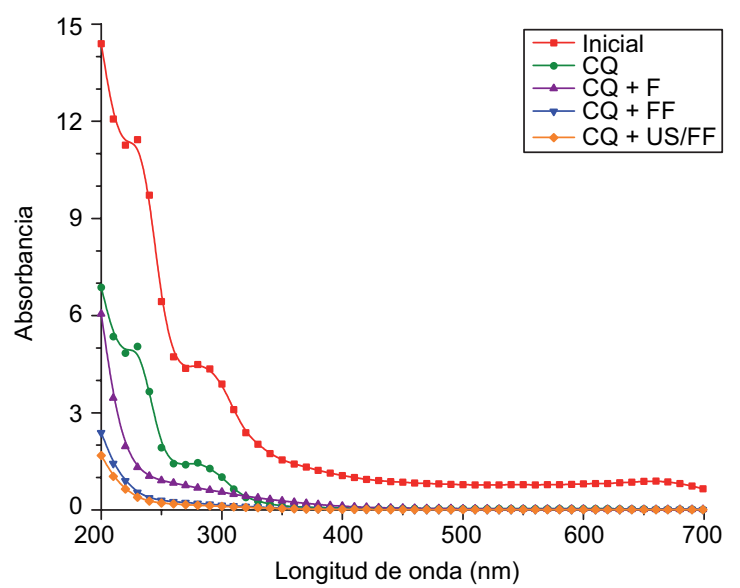

Fig. 5. Proceso secuencial coagulación química/ultrasonido/ Fenton-foto-Fenton (CQ/US/F-FF) para el tratamiento de aguas residuales textiles. (a) Degradación de la demanda química de oxígeno (\% DDQO) y agotamiento de $\mathrm{H}_{2} \mathrm{O}_{2}$. (b) Degradación del carbono orgánico total (\% DCOT).

(c) Espectrofotometría ultravioleta/visible orgánicos, detergentes y otros agentes químicos usados en la fabricación de textiles. En la figura 5c se observa que la muestra resultante del proceso de CQ no presenta absorbancia en el espectro visible (350$700 \mathrm{~nm}$ ), debido a que los colorantes se encuentran suspendidos y son susceptibles de desestabilización y remoción en la etapa de CQ. El proceso US/FF es el más eficiente; alcanza un \% DCOT de $78 \%$ después de 90 min de reacción, en tanto que el US/F logra $63 \%$ de mineralización. En ambos casos se obtuvieron mayores eficiencias en presencia de ondas US que en su ausencia. Estos resultados concuerdan con diversas investigaciones en las que se ha demostrado la capacidad del US para incrementar la eficiencia de los procesos Fenton. Ammar (2016) encontró que al combinar US de $20 \mathrm{kHz}$ con el proceso $\mathrm{F}$ se incrementó la velocidad de eliminación del contaminante en casi $20 \%$ a los 90 min de reacción. Por otro lado, Hou et al. (2016) emplearon el proceso $\mathrm{F}$ en fase heterogénea acoplado a US de frecuencia de $20 \mathrm{kHz}$ para el tratamiento de aguas contaminadas con tetraciclina, y encontraron que la eficiencia del proceso se incrementó, aproximadamente el $25 \%$ al utilizar el acoplamiento.

\section{CONCLUSIONES}

Los procesos Fenton y foto-Fenton acoplados a ultrasonido y coagulación química para el tratamiento de aguas residuales en la industria textil son eficientes para la remoción y degradación de los contaminantes presentes. A partir de los resultados de la investigación se plantean las siguientes conclusiones:

1. El pretratamiento de coagulación química es útil para la remoción de todos los sólidos suspendidos en el agua residual, y éstos representan alrededor de $50 \%$ de la materia orgánica presente en la solución.

2. Con la MSR-DBB se estableció el efecto de cada una de las variables de operación sobre la degradación de la DQO, resultando estadísticamente significativas. Valores de $\mathrm{pH}$ mayores que 3 causaron una reducción de la $\left[\mathrm{Fe}^{2+}\right]$ en la solución, aun en presencia de cavitación acústica, disminuyendo la eficiencia del proceso. Incrementos tanto en la $\left[\mathrm{Fe}^{2+}\right]$ como en la $\left[\mathrm{H}_{2} \mathrm{O}_{2}\right]$ promovieron un aumento en la eficiencia del proceso en el intervalo evaluado.

3. Mediante la utilización del modelo matemático de segundo orden se establecieron las condiciones óptimas del proceso $\mathrm{F}$ acoplado a US (F/US), las cuales fueron: $1 \mathrm{mM}$ de $\mathrm{Fe}^{2+}, 14 \mathrm{mM}$ de $\mathrm{H}_{2} \mathrm{O}_{2}$ y 
$\mathrm{pH} 3$, con coeficientes de determinación $\mathrm{R}^{2} \mathrm{y} \mathrm{R}_{\text {adj }}^{2}$ de 94.4 y $84.2 \%$, respectivamente.

4. La cinética de degradación evidencia que al acoplar el US con los procesos $\mathrm{F}$ y FF se incrementa la eficiencia del proceso en alrededor de $10 \%$, tanto para el \% de DDQO como para el \% de DCOT, en 90 min de reacción. La cavitación acústica se considera como una tecnología viable para la intensificación de la eficiencia en los procesos Fenton.

\section{AGRADECIMIENTOS}

Este trabajo se desarrolló con el apoyo de la Dirección de investigación de la Universidad Escuela de Administración Finanzas y Tecnología (EAFIT) y del Departamento Administrativo de Ciencias, Tecnología e Innovación (COLCIENCIAS), programa Jóvenes Investigadores.

\section{REFERENCIAS}

Ammar H.B. (2016). Sono-Fenton process for metronidazole degradation in aqueous solution: Effect of acoustic cavitation and peroxydisulfate anion. Ultrason. Sonochem. 33, 164-169.

DOI: $10.1016 /$ j.ultsonch.2016.04.035

APHA (2012). Standard methods for the examination of water and wastewater. 22a ed. American Public Health Association. Washington, $724 \mathrm{pp}$.

Amor C., Torres-Socías E., Peres J.A., Maldonado M.I., Oller I., Malato S. y Lucas M.S. (2015). Mature landfill leachate treatment by coagulation-flocculation combined with Fenton and solar photo-Fenton processes. J. Hazard. Mater. 286, 261-268.

DOI: $10.1016 /$ j.jhazmat.2014.12.036

Arslan-Alaton I., Tureli G. y Olmez-Hanci T. (2009). Treatment of azo dye production wastewaters using Photo-Fenton-like advanced oxidation processes: Optimization by response surface methodology. J. Photochem. Photobiol. A 202 (2-3), 142-153.

DOI: 10.1016/j.jphotochem.2008.11.019

Bagal M.V. y Gogate P.R. (2014). Wastewater treatment using hybrid treatment schemes based on cavitation and Fenton chemistry: A review. Ultrason. Sonochem. $21(1), 1-14$.

DOI: 10.1016/j.ultsonch.2013.07.009

Banerjee P., Chakrabarti S., Maitra S. y Dutta B.K. (2012). Zinc oxide nano-particles - sonochemical synthesis, characterization and application for photoremediation of heavy metal. Ultrason. Sonochem. 19 (1), 85-93. DOI: 10.1016/j.ultsonch.2011.05.007
Blanco J., Torrades F., Morón M., Brouta M. y García J. (2014). Photo-Fenton and sequencing batch reactor coupled to photo-Fenton processes for textile wastewater reclamation: Feasibility of reuse in dyeing processes. Chem. Eng. J. 240, 469-475.

DOI: $10.1016 /$ j.cej.2013.10.101

Buscio V., Marín M.J., Crespi M. y Gutiérrez-Bouzán C. (2015). Reuse of textile wastewater after homogenization-decantation treatment coupled to PVDF ultrafiltration membranes. Chem. Eng. J. 265, 122-128. DOI: $10.1016 /$ j.cej.2014.12.057

Cai M., Su J., Lian G., Wei X., Dong C., Zhang H., Jin M. y Wei Z. (2016). Sono-advanced Fenton decolorization of azo dye Orange G: Analysis of synergistic effect and mechanisms. Ultrason. Sonochem. 31, 193-200. DOI: 10.1016/j.ultsonch.2015.12.017

Carvalho J.C., Bessegato G.G. y Zanoni M.V. (2016). Efficiency comparison of ozonation, photolysis, photocatalysis and photoelectrocatalysis methods in real textile wastewater decolorization. Water Res. 98, 39-46. DOI: 10.1016/j.watres.2016.04.004

Cheshmeh R.D. y Safari M. (2016). Periodate-assisted pulsed sonocatalysis of real textile wastewater in the presence of $\mathrm{MgO}$ nanoparticles: Response surface methodological optimization. Ultrason. Sonochem. 32, 181-190.

DOI: 10.1016/j.ultsonch.2016.03.011

Dias F.F., Oliveira A.S., Arcanjo A.P., Moura F.C. y Pacheco J.C. (2016). Residue-based iron catalyst for the degradation of textile dye via heterogeneous photoFenton. Appl. Catal. B. 186, 136-142.

DOI: $10.1016 /$ j.apcatb.2015.12.049

Doumic L.I., Soares P.A., Ayude M.A., Cassanello M., Boaventura R. y Vilar V. (2015). Enhancement of a solar photo-Fenton reaction by using ferrioxalate complexes for the treatment of a synthetic cotton-textile dyeing wastewater. Chem. Eng. J. 277, 86-96.

DOI: $10.1016 /$ j.cej.2015.04.074

Gernjak W., Fuerhacker M., Fernández-Ibañez P., Blanco J. y Malato S. (2006). Solar photo-Fenton treatmentProcess parameters and process control. Appl. Catal. B. 64 (1-2), 121-130. DOI: 10.1016/j.apcatb.2005.12.002

GilPavas E., Medina J., Gómez-García M. y Dobrosz I. (2014). Statistical optimization of industrial textile wastewater treatment by electrochemical methods. J. Appl. Electrochem. 44 (12), 1421-1430.

DOI: $10.1007 / \mathrm{s} 10800-014-0767$

GilPavas E., Gómez C., Gómez-García M. y Dobrosz I. (2015). Decolorization and mineralization of Yellow 5 (E102) by UV/ $/ \mathrm{Fe}^{2+} / \mathrm{H}_{2} \mathrm{O}_{2}$ process. Optimization of the operational conditions by Response Surface Methodology. C. R. Chim. 18 (10), 1152-1160.

DOI: $10.1016 /$ j.crci.2015.08.001 
GilPavas E., Dobrosz-Gómez I. y Gómez-García M. (2017). Coagulation-flocculation sequential with Fenton or photo-Fenton processes as an alternative for the industrial textile wastewater treatment. J. Environ. Manage. 191, 189-197.

DOI: 10.1016/j.jenvman.2017.01.015

Hou L., Wang L., Royer S. y Zhang H. (2016). Ultrasoundassisted heterogeneous Fenton-like degradation of tetracycline over a magnetite catalyst. J. Hazard. Mater. 302, 458-467. DOI: 10.1016/j.jhazmat.2015.09.033

Jorfi S., Barzegar G., Ahmadi M., Cheshmeh R., Jafarzadeh N., Takdastan A., Saeedi R. y Abtahi M. (2016). Enhanced coagulation-photocatalytic treatment of Acid red 73 dye and real textile wastewater using UVA/ synthesized $\mathrm{MgO}$ nanoparticles. J. Environ. Manage. 177, 111-118. DOI: 10.1016/j.jenvman.2016.04.005

Malato S., Fernández-Ibáñez P., Maldonado M.I., Blanco J. y Gernjak W. (2009). Decontamination and disinfection of water by solar photocatalysis: Recent overview and trends. Catal. Today 147 (1), 1-59.

DOI: $10.1016 /$ j.cattod.2009.06.018

Miralles-Cuevas S., Oller I., Sánchez-Pérez J.A., Malato S. (2014). Removal of pharmaceuticals from MWTP effluent by nanofiltration and solar photo-Fenton using two different iron complexes at neutral $\mathrm{pH}$. Water Res. 64, 23-31. DOI: 10.1016/j.watres.2014.06.032

Montgomery D.C. (2005). Response surface methods and designs. En: Design and analysis of experiments. John Wiley and Sons, Nueva York, pp. 427-492.

Naje A.S., Chelliapan S., Zakaria Z. y Abbas S. (2016). Electrocoagulation using a rotated anode: A novel reactor design for textile wastewater treatment. J. Environ. Manage. 176, 34-44.

DOI: $10.1016 /$ j.jenvman.2016.03.034

Papoutsakis S., Miralles-Cuevas S., Gondrexon N., Baup S., Malato S. y Pulgarin C. (2015). Coupling between high-frequency ultrasound and solar photo-Fenton at pilot scale for the treatment of organic contaminants: An initial approach. Ultrason. Sonochem. 22, 527-534. DOI: 10.1016/j.ultsonch.2014.05.003

Rodríguez M., Sarría V., Esplugas S. y Pulgarin C. (2002). Photo-Fenton treatment of a biorecalcitrant wastewater generated in textile activities: Biodegradability of the phototreated solution. J. Photochem. Photobiol. A 151 (1-3), 129-135.

DOI: 10.1016/S1010-6030(02)00148-X

San Pedro-Cedillo L., Méndez-Novelo R.I., Rojas-Valencia M.N., Barceló-Quintal M., Castillo-Borges E.R., Sauri-Riancho M.R. y Marrufo-Gómez J.M. (2015). Evaluation of adsorption and Fenton-adsorption processes for landfill leachate treatment. Rev. Mex. Ing. Quim. 14 (3), 745-755.

DOI: $10.9734 / \mathrm{BJAST} / 2016 / 28051$

Sheydaei M. y Khataee A. (2015). Sonocatalytic decolorization of textile wastewater using synthesized c-FeOOH nanoparticles. Ultrason. Sonochem. 27, 616-622.

DOI: 10.1016/j.ultsonch.2015.04.023

Silva N.A., Martins R.C., Paiva C., Castro-Silva S. y Quinta-Ferreira R. (2016). A new winery wastewater treatment approach during vintage periods integrating ferric coagulation, Fenton reaction and activated sludge. J. Environ. Chem. Eng. 4 (2), 2207-2215.

DOI: $10.1016 /$ j.jece.2016.03.044

Vedrenne M., Vásquez R., Prato-García D., Frontana B.A. e Ibánez J.G. (2012). Characterization and detoxification of a mature landfill leachate using a combined coagulation-flocculation/photo Fenton treatment. J. Hazard. Mater. 205-206, 208- 215.

DOI: 10.1016/j.jhazmat.2011.12.060

Verma A.K., Dash R.R. y Bhunia P. (2012). A review on chemical coagulation/flocculation technologies for removal of colour from textile wastewaters. J. Environ. Manage. 93 (1), 154-168.

DOI: $10.1016 /$ j.jenvman.2011.09.012

Wang C. y Shih Y. (2015). Degradation and detoxification of diazinon by sono-Fenton and sono-Fenton-like processes. Sep. Purif. Technol. 140, 6-12.

DOI: 10.1016/j.seppur.2014.11.005

Yurtsever A., Cınar Ö. y Sahinkaya E. (2016). Treatment of textile wastewater using sequential sulfate-reducing anaerobic and sulfide-oxidizing aerobic membrane bioreactors. J. Membr. Sci. 511, 228-237.

DOI: 10.1016/j.memsci.2016.03.044 